\title{
THE IMPORTANCE OF LOADING FREQUENCY, RATE AND VIBRATION FOR ENHANCING BONE ADAPTATION AND IMPLANT OSSEOINTEGRATION
}

\author{
A. Torcasio, G.H. van Lenthe, and H. Van Oosterwyck* \\ Division of Biomechanics and Engineering Design, K.U. Leuven, Leuven, Belgium
}

\begin{abstract}
Mechanical loading is one of the key factors that influence bone mass and the osseointegration of bone-anchored implants. From a clinical point of view, mechanical stimulation may be used to enhance bone strength and implant osseointegration. Among the many loading parameters that influence the response to mechanical loading, the effects of loading frequency and rate have been investigated in many studies. In this paper the most relevant animal studies that have addressed the effect of loading frequency, rate, and vibration on either bone adaptation or implant osseointegration are systematically reviewed. Apparently contradictory results are discussed and interpreted within the context of mechanotransduction and mechanoregulation of bone. A combined experimental and computational approach is suggested to address some of the remaining research questions.
\end{abstract}

Keywords: bone adaptation, loading frequency, strain rate, vibration, osseointegration, implant.

*Address for correspondence:

Hans Van Oosterwyck

Division of Biomechanics and Engineering Design, K.U. Leuven

Celestijnenlaan 300C, PB 2419

B-3001 Leuven, Belgium

Telephone Number: +32 16327067

FAX Number: +32 16327994

E-mail: hans.vanoosterwyck@mech.kuleuven.be url: http://www.mech.kuleuven.be/bmgo/

\section{Introduction}

Bone as a living tissue has long been recognized to be capable of adapting its mass and structure in response to the demands of mechanical loading. The ability of bone to respond to mechanical signals has been repeatedly demonstrated in the past (Burr et al., 1985; Lanyon and Rubin, 1984; Turner et al., 1994) and also our understanding of how these signals are converted into an anabolic or catabolic response has increased considerably. Osteocytes, buried in the bone matrix, and the lacunocanalicular porosity are believed to be the professional mechanosensory cells of bone and the structure that mediates mechanosensing respectively (Burger and KleinNulend, 1999; Cowin, 2002; Turner et al., 1994; You et al., 2001). The external mechanical stimuli are transduced into a cellular response through a process known as "mechanotransduction". Due to the difficulty of directly measuring the mechanisms in vivo, hypotheses on how bone may sense mechanical loading are to a large extent based on mathematical models. Physiological loading results in bone matrix deformation and creates pressure gradients within the fluid-filled lacunar-canalicular network, giving rise to the movement of extracellular fluid and osteocyte deformation. The (micro)mechanics by which fluid movement is coupled to cellular deformation can be quite complex and have been studied by means of mathematical models, such as the one from You et al. (2001). This model considers an osteocyte process in a canaliculus and its pericellular matrix that fills the space between the canalicular wall and the membrane of the cellular process. The model predicts that load-induced fluid flow leads to shear forces on the plasma membrane of the cellular process and drag forces on the fibrils in the pericellular matrix. Interestingly, the model also suggests that tissue strains may be amplified at the level of the cellular processes due to their microstructural organisation, providing further evidence of the importance of the osteocytes as the mechano-sensitive cells par excellence. If bone adaptation is driven by fluid flow, then adaptation should occur only in response to dynamic and not to static loads (Jacobs et al., 1998), as the latter will not create fluid movement. Indeed, this was already noticed by Liskova and Hert (1971) in rabbits, which was later confirmed by Lanyon and Rubin (1984) by means of their well known isolated avian ulna model.

At a more phenomenological level, several mechanical parameters have been identified that influence the anabolic response to loading. Among these are strain rate, number of cycles (Kaspar et al., 2002), strain distribution, local strain gradients (Judex et al., 1997), and resting periods (LaMothe and Zernicke, 2004; Srinivasan et al., 2002). Strain rate can be further decomposed into strain magnitude and loading frequency (see below for a more detailed explanation), suggesting that low-magnitude 
high-frequency mechanical stimuli may be as stimulatory as high magnitude low-frequency stimuli (Bacabac et al., 2004). Low amplitude high frequency loading in bone has been shown to occur more often in normal daily activities in vivo (Fritton et al., 2000) and might be used to promote adaptive bone formation.

The tissue's sensitivity to functional loading suggests an interesting potential pathway for therapeutic intervention. Mechanical stimuli could be capable of inhibiting or even reversing bone loss caused by diseases such as osteoporosis (Rubin et al., 2001a; Rubin et al., $2002 b)$. It has also been postulated that the adaptive capacity of bone in response to specific loading regimes could be considered a means by which biological fixation of prostheses could be promoted. In contrast, unfavourable conditions of reduced load transfer at the bone-implant interface can initiate significant bone resorption ('stress shielding') and may contribute to eventual degradation of the long-term implant performance (Huiskes et al., 1992; Pilliar et al., 1979). In addition, both animal experimental and computational studies have demonstrated the importance of mechanical loading for the early healing response around implants (Duyck et al., 2006; Geris et al., 2008; Huiskes et al., 1997; Prendergast et al., 1997; Søballe et al., 1992; Vandamme et al., 2007). Similar to fracture healing, peri-implant tissue differentiation seems to be mediated by mechanics. Again, one could think of creating a mechanical environment that favours the differentiation towards bone, in order to accelerate implant osseointegration.

This paper is aimed at evaluating the possibility of using mechanical stimuli to enhance quality and quantity of bone, without or within the presence of an implant. More specifically a literature review was performed on in vivo animal studies in which loading frequency and rate were systematically varied within the context of bone adaptation or implant osseointegration. In addition, we also included studies that have explored the use of mechanical vibration to enhance bone mass. For the interpretation of these studies it is important to have a basic understanding of the relationship between commonly used loading parameters. Therefore, some basic mathematical relationships between these loading parameters will be presented first.

\section{Mathematical relationships between strain rate, amplitude and frequency}

Strain rate, magnitude and loading frequency of a dynamic loading are interrelated (Turner, 1998). The relationships depend on the applied loading waveform (e.g. sinusoidal or trapezoidal). These concepts will be further elaborated below.

In an experimental system in which a bone is subjected to both stationary and cyclic loads, the applied load is given by:

$$
\mathrm{F}(\mathrm{t})=\mathrm{F}_{0}+\mathrm{F}_{1} \sin \omega \mathrm{t}
$$

where $\mathrm{F}_{0}$ is the magnitude of the stationary load, $\mathrm{F}_{1}$ is the amplitude of the cyclic load, $\omega$ is the angular frequency ( $\omega=2 \pi f$, where $f$ is the loading frequency) and $t$ is the time. Considering bone tissue to be a linear elastic material, the local strain, encountered at a given location in the bone tissue, will follow a similar time-dependency:

$$
\varepsilon(\mathrm{t}, \mathrm{x})=\varepsilon_{0}(\mathrm{x})+\varepsilon_{1}(\mathrm{x}) \sin \omega \mathrm{t}
$$

where $\varepsilon(t, x)$ is the strain tensor (accounting for different normal and shear strain components), $\mathrm{x}$ is a vector that gives the position of a point inside the bone tissue, and $\varepsilon_{0}$ and $\varepsilon_{1}$ are again the stationary and dynamic component of the strain tensor. For a simple geometry and loading condition the relation between the external load and the strain may be solved analytically. For complex geometries like bones, numerical techniques like the finite element (FE) method are needed to calculate detailed strain distributions. Examples for the study of well-controlled loading setups can be found in Gross et al. (2002) and Kotha et al. (2004) for cantilever-like bending of a mouse tibia and axial compression of a rat ulna respectively. Experimental validation of these numerical models is typically done by means of strain gauge measurements on the cortical bone surface. In addition, strain gauge measurements are widely used to calibrate an experimental set-up, i.e. to derive an empirical relation between the external load and the bone surface strain at a specific location (see e.g., Rubin and Lanyon, 1985 and Warden and Turner, 2004). Obviously, unlike an FE model, this only provides information at the point of strain gauge attachment, and only in the direction of the strain gauge element (at least for single element strain gauges).

Once the dynamic strain (strain as a function of time) is known the strain rate $\varepsilon$ can be obtained by taking the time derivative of (2). For the sake of simplicity we will only consider here one strain component at a certain bone location (as e.g., measured by a single element strain gauge), leaving out the directional and spatial dependency:

$$
\dot{\varepsilon}(\mathrm{t})=\frac{\partial \varepsilon}{\partial \mathrm{t}}=\omega \varepsilon_{1} \cos \omega \mathrm{t}=2 \pi \mathrm{f} \varepsilon_{1} \cos \omega \mathrm{t}
$$

In which $\mathrm{f}$ is the loading frequency and $\varepsilon_{1}$ is the amplitude of the dynamic strain component (now a scalar value). Based on the experimental observations that bone adaptation seems to increase with increasing values of the dynamic strain amplitude (further denoted as $\varepsilon$ instead of $\varepsilon_{1}$ ) and loading frequency, Turner proposes the following governing stimulus (Turner, 1998):

$$
\mathrm{E}=\mathrm{k}_{1} \varepsilon f
$$

$\mathrm{E}$ is called the strain stimulus and $\mathrm{k}_{1}$ is proportionality constant. It must be noted that this stimulus is not equal to strain rate (as this is a function of time as well, see (3)), but it is proportional to the amplitude of strain rate (which is equal to $2 \pi f \varepsilon_{1}$ according to (3)).

In his review paper Turner proposes a generalisation of this stimulus for non-sinusoidal periodic loading conditions. Indeed, any kind of period function can be expanded to a Fourier series, which is an (infinite) sum of sines and cosines. As an example the Fourier series expansion of a trapezoidal loading waveform is given in figure 1.

In practice a finite number of terms is needed to obtain an approximation of the periodic waveform. Similar to an 


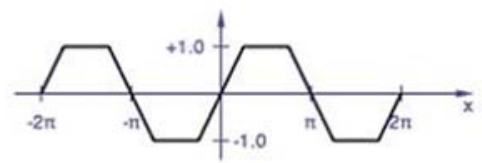

$$
F_{\text {trap }}(t)=\frac{8}{\pi^{2}} \sum_{n=1}^{\infty} \frac{\sin \frac{n \pi}{4}+\sin \frac{3 n \pi}{4}}{n^{2}} \sin n t
$$

Figure 1: Fourier series expansion of a trapezoidal loading waveform (trapezoidal waveform normalised to one, periodic on $[-\pi, \pi]$ ) (taken from http://www.till.com/articles/QuadTrapVCO/trapezoid.html)

arbitrary periodic loading wave, the corresponding strain and strain rate can be written by means of a (finite) series expansion:

$$
\begin{aligned}
& \varepsilon(t)=\sum_{n=1}^{k} \varepsilon_{n} \sin n t=\sum_{n=1}^{k} \varepsilon_{n} \sin 2 \pi f_{n} t \\
& \dot{\varepsilon}(t)=\sum_{n=1}^{k} \varepsilon_{n} n \cos n t=2 \pi \sum_{n=1}^{k} f_{n} \varepsilon_{n} \cos 2 \pi f_{n} t
\end{aligned}
$$

With $\varepsilon_{\mathrm{n}}$ and $\mathrm{f}_{\mathrm{n}}=\mathrm{n} / 2 \pi$ being the amplitude and frequency of the $n$-th term respectively in the expansion of the strain wave. Similar to a single sine-loading wave, Turner proposes that in the case of any periodic loading condition the strain stimulus can be defined in terms of the amplitudes and frequencies of the different terms (components) of the strain:

$$
\mathrm{E}=\mathrm{k}_{1} \sum_{\mathrm{n}=1}^{\mathrm{k}} \varepsilon_{\mathrm{n}} \mathrm{f}_{\mathrm{n}}
$$

Again, it must be noticed that this stimulus is different from strain rate. Instead, it is proportional to the sum of the strain rate amplitudes of the different components of the strain rate signal. This difference becomes important when experiments that apply either sinusoidal or nonsinusoidal (e.g. trapezoidal) periodic loading waves are interpreted. For a single sine wave the strain signal has only one frequency component, and therefore there will be only one term in the strain stimulus, as defined by (7). On the other hand, the strain rate is varying as a function of time, as it is a cosine function (equation (3). For a trapezoidal loading wave a series of frequency components (frequency spectrum) is retrieved, leading to as many contributions to the strain stimulus in (7). On the other hand, the strain rate during loading and unloading is proportional to the slope of the loading wave (figure 1) and is therefore constant. In addition, as already mentioned by Turner (1998) sinusoidal loading allows to vary the loading amplitude, while keeping frequency constant. At the same time, strain rate (equation (3)) as well as the strain stimulus (equation (4)) will be varied as well. For a trapezoidal loading wave, a change of the height of the trapezoid (and therefore strain magnitude) can be obtained without changing the loading (and strain) rate, as long as the slope of the trapezoid remains unchanged. This was e.g. the case in the experiments by Rubin and Lanyon (1985) and Mosley et al. (1997). However, changing the height will lead to a different frequency spectrum and therefore a different value of the strain stimulus, defined in equation (7).

\section{Bone adaptation studies}

The effects of loading frequency and rate have been assessed in several animal studies. We can distinguish between experiments in which individual limbs/bones of the animal are exposed to mechanical loading and "Whole Body Vibration" in which the body or large part thereof of the animal under investigation is mechanically stimulated by means of vibrating platforms. Loading of individual limbs provides much more control on the loading characteristics (magnitude, frequency, waveform...) to which bones are exposed. In many of these studies information is also provided on the strain magnitude on the (cortical) bone surface experienced during load application (either measured by strain gauges or calculated by means of FE modelling). Well-known examples are the isolated avian ulna model (Lanyon and Rubin, 1984), the rat (Torrance et al., 1994) and mouse (Lee et al., 2002) ulnar compression model and the mouse tibial compression model (De Souza et al., 2005), the latter three having the advantage of being non-invasive.

When using vibrating platforms, it is much less straightforward what is the mechanical stimulus (magnitude, frequency, waveform) to which the bones are exposed to. Studies that have used such devices often only provide information on the frequency and the maximum acceleration of the platform (see e.g. Flieger et al., 1998, Oxlund et al., 2003). Both loading parameters are coupled. Suppose a vibration motor results in a sinusoidal displacement $\mathrm{x}$ of the platform:

$$
x(t)=A \sin \omega t=A \sin (2 \pi f t)
$$

with $\mathrm{A}$ being the displacement amplitude, $\omega$ and $\mathrm{f}$ being the angular frequency and frequency respectively. The acceleration, being defined as the second derivative of the displacement is then given by:

$$
a(t)=-A \omega^{2} \sin \omega t=-4 A \pi^{2} f^{2} \sin (2 \pi f t)
$$

The maximum acceleration is proportional to the square of the frequency. Unless the vibration motor also allows changing the displacement amplitude A, both parameters cannot be varied independently. In addition, as force transfer through the limbs may be very complex and among other factors may involve soft tissue dampening and muscle activation, one cannot readily derive what are the loading (strain) magnitude and frequency at the level of the bones (unless they are measured by strain gauges, see e.g., Rubin et al., 2002a, 2002b).

Obviously, this complicates the comparison between loading studies on individual limbs and vibration studies. Another difference lies in the order of applied strains: typically, studies on individual limbs have investigated the effect of mechanical stimulation for strains that are orders of magnitude larger than for studies with vibrating platform (typically of the order of 100-1000 $\mu \varepsilon$ versus $1-10 \mu \varepsilon$ respectively). 


\section{Loading studies on individual limbs}

Following our discussion on the relation between loading rate, frequency and amplitude we make a distinction here between sinusoidal (as e.g., used by Turner and coworkers) and trapezoizal (as e.g., used by Lanyon and coworkers). While the first loading wave is suitable for exploring the effect of loading frequency and amplitude independently (and within this context the role of the strain stimulus, defined in equation (4)), the second one allows applying a single strain rate value.

\section{Sinuoidal loading}

Turner et al. (1994) measured the effect of applying sine waves at different loading frequencies $(0.05,0.1,0.2,0.5$, 1 and $2 \mathrm{~Hz}$ ) of the same magnitude $(52 \mathrm{~N})$ for 36 cycles per day on cortical bone formation rate in the right tibiae of rats. Either bending (through a four-point bending apparatus) or axial loading was applied as a sine wave. Histomorphometric indices (Parfitt et al., 1987) on the periosteal surface in the mid-diaphyseal region of the left tibia were obtained and were expressed as relative values with respect to the contralateral unloaded limb. The analysis revealed that axial loading did not cause a significant increase in the relative bone formation rate over the bone surface (rBFR/BS) but that bending increased rBFR/BS for loading frequencies between 0.5 and $2 \mathrm{~Hz}$. An explanation for this finding can be that for axial loading the induced strains were lower and less anabolic than for bending.

The anabolic response to bending was further explored for the same animal model, by varying the load magnitude $(0,18,36$ and $54 \mathrm{~N})$ and keeping the frequency constant ( $2 \mathrm{~Hz}$ ) (Turner et al., 1995). They found that relative bone formation rate (rBFR) on the endocortical surface was proportional to load magnitude. By combining the bending results of both studies and plotting rBFR with respect to the strain stimulus (equation (4)), Turner (1998) found similar linear relations for both studies, therefore providing evidence for the strain stimulus being the governing stimulus (at least for frequencies up to $2 \mathrm{~Hz}$ ).

Hsieh and Turner (2001) further examined the validity of the strain stimulus for frequencies equal to 1,5 , or 10 Hz. A sinusoidal compressive loading was applied on the right ulna of rats for 360 cycles/day for 10 days with peak loads ranging from 4.3 to $18 \mathrm{~N}$. They found that the relative periosteal bone formation rate (given by $\mathrm{rBFR} / \mathrm{BS}$ ) was proportional to peak strain at each of the three loading frequencies tested. The proportionality was different for the three test groups, with similar strains being the most anabolic at $5 \mathrm{~Hz}$ and the least anabolic at $1 \mathrm{~Hz}$. When plotting periosteal bone formation rate against the strain stimulus (equation 4) regression analysis revealed two distinct curves, one for the $1 \mathrm{~Hz}$ test results and one for the combined 5 and $10 \mathrm{~Hz}$ results. Using the same ulnaloading model (but adapted to the mouse), Warden and Turner (2004) studied bone adaptation to sinusoidal load with frequency of $1,5,10,20$ and $30 \mathrm{~Hz}$ for C57BL/6 mice. The histomorphometry response of the right ulna was studied when loaded at one of the five frequencies and one of two load magnitudes (1.5 and $2 \mathrm{~N}$ ). Relative bone formation rate $(\mathrm{rBFR} / \mathrm{BS})$ in the periosteal surface at each frequency and peak load revealed that there were no differences among frequencies when loading at $1.5 \mathrm{~N}$. However, when loading at $2 \mathrm{~N}$, cortical bone adaptation increased with increasing loading frequency up to 5-10 $\mathrm{Hz}$. No additional bone adaptation with loading frequencies beyond $10 \mathrm{~Hz}$ was found. Similar to the previous study, it reveals that the proposed strain stimulus, which accounts for strain amplitude and frequency, is not able to fully describe the response to mechanical loading.

Recently, Castillo et al. (2006) investigated the effect of low-amplitude broad-frequency vibration, superimposed on a $2 \mathrm{~Hz}$ sinusoidal loading wave, for the same mouse ulnar compression model (again for C57BL/ 6 mice). Two studies were completed wherein several loading waveforms, with or without an additive lowamplitude, broad-frequency $(0-50 \mathrm{~Hz})$ vibration signal, were applied to the right ulnae. In a first study, mice were loaded short-term ( $30 \mathrm{~s} /$ day, 2 days) with either a sine wave signal alone $(1$ or $2 \mathrm{~N})$, vibration signal alone $(0.1 \mathrm{~N}$ or $0.3 \mathrm{~N}$ ) or combined sinusoidal and vibration signal. In a second study, mice were loaded long-term (30 s/day, 3 days/week, 4 weeks) with a sinusoidal signal alone, static signal alone, vibration signal alone $(0.02 \mathrm{~N}, 0.04 \mathrm{~N}, 0.08$ $\mathrm{N}$ or $0.25 \mathrm{~N}$ ) or combined sinusoidal and vibration signal. After loading, cortical bone formation parameters were assessed by histomorphometry. Data from the first study indicated that vibration increased cortical bone formation when superimposed to the sine wave. Animals that were loaded with the $2 \mathrm{~N}$ sine waveform alone plus $0.3 \mathrm{~N}$ vibration signal had significantly greater bone formation rates than animals loaded with the $2 \mathrm{~N}$ sine wave alone. However, further review of these data indicated that the enhanced response could be explained by increased load and strain levels rather than by the vibration signal itself. This confounding factor may also explain the enhanced osteogenic response for a similar combined waveform, reported by Tanaka et al. (2003). Also in this study broad frequency noise was added to a $2 \mathrm{~Hz}$ sine wave, without compensating for the load and strain magnitude. In the second study of Castillo et al. (2006) the magnitude of the sinusoidal waveform was reduced to counterbalance the increase in magnitude introduced by the superimposed vibration signal. Data from the second study showed that cortical bone formation was not significantly altered by vibration. These findings suggested that low-amplitude, broad-frequency vibration superimposed onto an osteogenic waveform or vibration alone did not enhance cortical bone adaptation at the frequencies, amplitudes and loading periods tested.

\section{Trapezoidal loading}

Mosley and Lanyon (1998) used a trapezoidal loading waveform to test the anabolic potential of the strain rate by applying an axial compression load between 1 and 20 $\mathrm{N}$ on the left ulna of Sprague Dawley rats, corresponding to about $4000 \mu \varepsilon$ at the medial midshaft (compressive strain). Three levels of strain rate (i.e. slope during loading and unloading) were tested: low $( \pm 0.018 / \mathrm{sec})$, moderate $( \pm 0.030 / \mathrm{sec})$ and high $( \pm 0.100 / \mathrm{sec})$. The frequency (referring here to the number of trapezoidal cycles per second, which is different from the frequency spectrum, 
as retrieved from a Fourier series expansion, see fig. 1) of $2 \mathrm{~Hz}$ was kept constant by altering the low dwell period ('rest period') between trapezoids. Periosteal bone formation was evaluated at different positions along the midshaft and compared to the contralateral (nonstimulated) right ulna. For all three test groups a similar site-specific response was found, with the highest loadrelated osteogenic response (compared to contralateral control) at 2-3 $\mathrm{mm}$ distal from the midshaft. When comparing test groups, the highest osteogenic response was found for the highest strain rate group, the lowest for the lowest strain rate group. The positive effect of the insertion of the rest periods in affecting the potential of the mechanical loading was not taken into account. Although this has been shown to have an effect in other studies (Robling et al., 2001; Srinivasan et al., 2002), it may not have been relevant for this study due to the short (less than half a second) rest periods.

LaMothe et al. (2005) tested the hypothesis that high strain rate enhanced the adaptive response by applying a 1 $\mathrm{Hz}$ trapezoidal waveform for $60 \mathrm{~s}, 5$ days per week, for 4 weeks in $\mathrm{C} 57 \mathrm{BL} / 6$ mice. The proximal right tibia was immobilized, and loads were applied to the distal lateral tibia so that the tibia was in cantilever bending. Unlike Mosley and Lanyon (1998), the frequency (again defined as number of trapezoidal cycles per second) was kept constant by altering the high dwell period (i.e., period of maximum load). Relative mineral apposition rate and bone formation rate were evaluated for three different levels of strain rate $(0.004 / \mathrm{s}, 0.02 / \mathrm{s}$, and $0.100 / \mathrm{s})$ on the periosteal and endocortical surface. For the three test groups significant increases (with respect to contralateral control) for almost all histomorphometrical parameters were found. The difference between the test groups was less pronounced, although for some of the parameters the highest strain rate led to a significantly higher increase than the other two strain rates.

\section{Vibration studies}

Experiments performed on sheep (Rubin et al., 2001a; Rubin et al., 2002a; Rubin et al., 2002b), rats (Flieger et al., 1998; Judex et al., 1997; Oxlund et al., 2003; Rubin et al., 2001b) and mice (Xie et al., 2006) tested the ability of high-frequency low-magnitude vibrations in promoting the anabolic response in trabecular and/or cortical bone.

Rubin et al. (2001, 2002a, 2002b) evaluated the hypothesis that extremely low level $(0.3 \mathrm{~g})$, high frequency $(30 \mathrm{~Hz})$ mechanical strains are anabolic. The hindlimbs of adult female sheep were stimulated for 20 minutes/day during one year, using a non-invasive vertical oscillation sufficient to induce approximately 5 microstrains (peakto-peak $30 \mathrm{~Hz}$ oscillations) on the cortex of the tibia (as measured by strain gauges). Femoral, tibial and (nonstimulated) radial bone was analysed by means of dual Xray absorptiometry (DXA), peripheral quantitative computer tomography (pQCT) and histomorpometry (Rubin et al., 2001a, 2001b, 2002a). In vivo DXA measurements revealed an increase of bone mineral density in the tibia (whole bone measurement) of test versus control group, which was significant only at week 29 . In order to rule out any systemic effects, the same measurements were also done on the distal radius, where no significant differences were found between test and control groups. Post mortem pQCT of the proximal femur revealed no differences in bone mineral density for the cortical bone, but a $34.2 \%$ increase in trabecular bone density in the test group. Histomorphometry on undecalcified sections of the same trabecular bone region revealed a $45 \%$ increase in trabecular number and a $36 \%$ decrease in trabecular spacing. An increase in bone formation rate and mineralising surface could also be detected, although these differences were much less significant. In contrast, no histomorphometrical differences between test and control group could be found in the trabecular bone of the distal radius, again ruling out any systemic effect. In addition trabecular bone from the distal femoral condyle was analysed by means of microfocus computer tomography $(\mu \mathrm{CT})$ for the same experiment (Rubin et al., 2002b). In the experimental animals bone mineral content was $10.6 \%$ greater, the trabecular number was $8.3 \%$ higher, while the trabecular spacing decreased with $11.3 \%$, indicating that the higher bone quantity was related to a higher number of trabeculae and a higher trabecular thickness. All together, these findings indicated that low magnitude high frequency vibrations can be effectively transmitted to the bone (at least to the level of cortical bone) and that they can enhance bone mass in trabecular bone in a locationdependent, non-systemic way. The results also demonstrate that the study outcome may depend on the analysed anatomical location (e.g. proximal versus distal femur) and the applied analysis techniques (e.g. DXA, pQCT, $\mu C T$ and histology), which may strongly vary in terms of their discriminative power.

Adult female Sprague-Dawley rats were used by Rubin et al. (2001b) in order to study the ability of extremely low-magnitude $(0.25 \mathrm{~g})$, high-frequency $(90 \mathrm{~Hz})$ whole body vibration to restore bone metabolism in animals, exposed to disuse (tail suspension). After 28 days, bone formation rate over bone volume (BFR/BV) and mineralizing surface over bone surface $(\mathrm{MS} / \mathrm{BS})$ in the trabecular bone of the proximal tibia increased for mechanically stimulated rats compared to controls (not subjected to vibration). In contrast, those parameters were found to decrease in the disuse group. Disuse interrupted by $10 \mathrm{~min}$ per day of vibration brought the histomorphometric parameters to normal values.

Similarly, Xie et al. (2006) investigated the effect of whole body vibration in the growing skeleton, by exposing 8 week old BALB/cByJ mice to whole body vibration at $45 \mathrm{~Hz}(0.3 \mathrm{~g})$ for $15 \mathrm{~min} /$ day during 3 weeks. In vivo strain gauging indicated that the mechanical signal induced 45 $\mathrm{Hz}$ strain oscillations of approximately 10 microstrain (peak-to-peak) on the periosteal surface of the proximal tibia, demonstrating that the high-frequency mechanical signal could be transmitted to the bone. Whole body vibration only affected bone formation rates (BFR/BS) on the endocortical surface of the metaphysis and osteoclastic resorption in metaphyseal and epiphysial trabecular bone, resulting in a respective increase and decrease with respect to age-matched controls. For all other histomorphometrical 
and chemical properties investigated no effect could be determined for either epiphyseal, metaphyseal and middiaphyseal bone.

In other studies (Flieger et al., 1998; Oxlund et al., 2003) the influence of low intensity whole body vibration on the prevention of bone loss due to postmenopausal osteoporosis in rats was investigated. Flieger et al. (1998) found that early bone loss after ovariectomy (OVX), as assessed by DXA, was prevented by stimulating the animals with low intensity vibrations (frequency $=50 \mathrm{~Hz}$, acceleration $=2 \mathrm{~g}$ ) for $30 \mathrm{~min} /$ day for 5 days/week. Whole body vibration led to significantly higher BMD values at 5 weeks after ovariectomy for both the femur and the tibia. No effect of vibration was found in sham-operated animals. Oxlund et al. (2003) used the ovariectomized (OVX) rat model to study the effect of whole body vibrations on bone mass and strength (tibia and femur) and muscle mass. The animals were subdivided in groups for evaluating the effect of vibration at different magnitudes and frequencies $(0.5 \mathrm{~g}$, $17 \mathrm{~Hz} ; 1.5 \mathrm{~g}, 30 \mathrm{~Hz} ; 3 \mathrm{~g}, 45 \mathrm{~Hz}$ ) given $30 \mathrm{~min} /$ day for 90 days. Histomorphometrical analyses of the tibia revealed a significantly higher periosteal mineral apposition rate for all vibration-stimulated OVX groups, while periosteal bone formation rate was only significantly higher for the $45 \mathrm{~Hz}$-stimulated OVX group (compared to non-stimulated OVX control group). Endocortical bone formation rates were not significantly different in OVX groups with or without vibration. Three-point bending of the tibia revealed no significant differences in stiffness and strength between the OVX test and control groups. Compression of the distal femoral metaphysis resulted in a significantly lower strength for all OVX groups (non-stimulated and stimulated) versus sham-operated controls. Only the 45 Hz-stimulated OVX group led to significantly higher strength compared to the non-stimulated OVX control group. No differences in muscle mass were seen for all test and control groups, indicating that vibration was likely to cause a direct mechanical effect on bone, rather than indirect through an increase of muscle mass. As maximum acceleration and frequency were coupled and changed at the same time (see equation 9) it cannot be concluded whether the highest response, observed for the $45 \mathrm{~Hz}$ stimulated OVX group, was related to the higher frequency or the higher acceleration and therefore potentially higher force transfer and engendered strains at the level of the bone. As to the latter, no attempt was made to measure cortical bone surface strains.

Judex et al. (2007) subjected OVX rats to whole body vibrations $(0.15 \mathrm{~g})$ at $45 \mathrm{~Hz}$ or $90 \mathrm{~Hz}$ for $10 \mathrm{~min} /$ day for 28 days and compared to OVX controls. In vivo strain gauge measurements were done on the proximal tibia. The $45 \mathrm{~Hz}$ regime resulted in an average strain of $2.12 \mu \varepsilon$ and an average peak strain rate of $312 \mu \varepsilon / \mathrm{s}$, while for the 90 $\mathrm{Hz}$ regime this resulted in $0.74 \mu \varepsilon$ and $194 \mu \varepsilon / \mathrm{s}$ respectively. Fast Fourier Transform (FFT) analysis revealed the presence of a dominant frequency component equal to the applied frequency. Bone formation rates in the metaphysis of the proximal tibia were significantly larger in the $90 \mathrm{~Hz}$ test group when compared to controls, while for the $45 \mathrm{~Hz}$ test group results were not different from the control group. This result was found for both trabecular and cortical bone. In addition, $\mu \mathrm{CT}$ analysis of the distal femur showed that $90 \mathrm{~Hz}$ stimulated rats had significantly more trabecular bone volume and thicker trabeculae in the epiphyseal, but not in the metaphyseal area (compared to control group and $45 \mathrm{~Hz}$ test group). The fact that the $90 \mathrm{~Hz}$ loading regime led to significantly lower strain and strain rate levels, suggests that for this experiment loading frequency was the dominating factor that determined the osteogenic response.

\section{Implant osseointegration studies}

While several studies have investigated the effect of loading frequency, rate and vibration on bone adaptation, few attempts have been made to address their effect on implant osseointegration. Rubin and McLeod (1994) used the turkey ulna model of disuse osteopenia to evaluate if mechanical loading could be used actively to stimulate bony ingrowth into a porous surface of a cylindrical implant placed across the dorsal and ventral cortices of the bone diaphysis. The relative bony ingrowth was quantified by backscatter electron microscopy after eight weeks of: disuse alone, disuse plus 100 seconds per day of a $1 \mathrm{~Hz}$ sinusoidal load, and disuse plus 100 seconds per day of a $20 \mathrm{~Hz}$ load. In this model mechanical loading of the tissues surrounding the implant was achieved by loading the host bone in bending, instead of applying a load directly to the implant. The strains generated in the cortex immediately adjacent to the implant were quantified by means of strain gauges and equalled $150 \mu \varepsilon$. The results showed that disuse alone caused loss of bone away from the implant surface, while a $1 \mathrm{~Hz}$ load regimen prevented resorption and stimulated some growth of bone into the implant. Finally the $20 \mathrm{~Hz}$ loading regime led to the most favourable response, with new bone being formed not only at the interface, but also at both the periosteal and endosteal surfaces.

De Smet and co-workers performed a number of studies on the effect of mechanical loading on implant osseointegration (De Smet et al., 2006, 2007). All studies were performed for bicortically fixed screw-type metallic implants that were directly loaded (in contrast with Rubin and McLeod, 1994) in bending by means of an eccentrically applied implant force. Implants were placed in the tibial diaphysis of the guinea pig, and the amount of peri-implant bone was quantified histologically and compared to contralateral (unstimulated) controls. Both amplitude and frequency were varied for sinusoidal loading waves, the main research hypothesis in all studies being that the peri-implant bone response is proportional to the strain stimulus, as defined in equation 4 (i.e. proportional to strain rate amplitude). In a first study the strain rate amplitude was varied between 1620 and $12000 \mu \varepsilon / \mathrm{s}$ (De Smet et al., 2006). In total 6 different sinusoidal loading regimes were evaluated ( 3 at $3 \mathrm{~Hz}, 3$ at $30 \mathrm{~Hz}$ ), with strain amplitudes varying between 160 and $1000 \mu \varepsilon$ (as measured by means of a strain gauge attached to the cortical bone at $1.3 \mathrm{~mm}$ from the implant neck). Loading started one week after implant installation at 1800 cycles/day, 5 days/week, for 4 weeks. After 4 weeks post implantation more peri- 
implant bone was seen for the mechanically stimulated implants with respect to control implants for all test groups, although the difference was not significant. The largest increase was seen for the lowest strain rate amplitude (1620 $\mu \varepsilon / \mathrm{s}$, corresponding to $3 \mathrm{~Hz}$ x $540 \mu \varepsilon$ ). When plotting the increase in bone surface at the distal medullary cavity (the area that exhibited the largest increase) with respect to strain rate amplitude, an inverse correlation was even obtained. Therefore, this study did not support the hypothesis that the peri-implant osteogenic response is governed by the strain rate amplitude. This finding was further substantiated in another study with the same animal model, but for a different screw-type implant (De Smet et al., 2007). Three different test groups were defined, all having the same strain rate amplitude of $1600 \mu \varepsilon / \mathrm{s}$, corresponding to a frequency of $3-10-30 \mathrm{~Hz}$ and a strain amplitude of 533-160-53 $\mu \varepsilon$ respectively. The load duration was taken equal to 10 minutes per day in all test groups. A significant increase in bone surface area (with respect to contralateral control) was only evident for the $3 \mathrm{~Hz}$-loading regime, while for the higher $(10-30 \mathrm{~Hz})$ frequency no differences could be found.

\section{Discussion}

\section{Loading studies on individual limbs}

Based on the findings of Hsieh and Turner (2001) and Warden and Turner (2004) it seems that the osteogenic response to dynamic loading is not proportional to the strain rate amplitude (equation 4) within a broad frequency range (1-30 Hz). Instead, a non-linear response was retrieved, with the highest response encountered at $5-10 \mathrm{~Hz}$ (Warden and Turner, 2004). One may speculate about the causes of this non-linearity. As in the mouse ulna loading model the applied force needs to be transferred through the soft tissues (skin, cartilage) of the joints, dampening may render the force transfer at higher frequencies less efficient, leading to lower strains for the same force magnitude. Indeed, Hsieh et al. (1999) found such an effect for compressive loading of the rat ulna and four point bending loading of the rat tibia, both loading models leading to a logarithmic decrease of strain (per unit of force amplitude) with increasing frequency. The authors contributed this to the viscoelastic properties of the soft tissues and concluded that these loading systems acted like a low-pass filter, which filtered out the higher frequency loading components. In contrast, Warden and Turner (2004) could not confirm this effect in the mouse ulna model. Their strain gauge measurements revealed a constant strain per unit of load for loading frequencies between 1 and $30 \mathrm{~Hz}$. Therefore, for their study dampening can be ruled out as a possible explanation.

Instead the authors believe that the non-linearity may be related to a non-linear mechanical response of one of the components of the mechanotransduction chain. Assuming that flow-induced deformation of osteocytes is the mechanism by which cells sense external loading, either the relation between bone matrix deformation and fluid flow or the relation between fluid flow and cell deformation (or both) may be frequency or rate dependent, leading to a less efficient mechanotransduction process above a certain frequency or rate threshold. This idea is not supported by the theoretical findings of You et al. (2001). Their model predicts that strains are amplified from the tissue to the cell level and that the strain amplification ratio (i.e., ratio of cellular strain versus tissue strain) is increased with increasing load frequency, suggesting a more efficient mechanotransduction process with increasing loading frequency. Although the increase of this ratio becomes less substantial for higher frequencies (up to $20 \mathrm{~Hz}$ ), they could not establish a decrease of the ratio beyond a certain frequency.

Apart from theoretical models, in vitro mechanotransduction experiments on bone cells may help to address the non-linearity observed by Turner and coworkers (obviously considering the limitations of in vitro models to explain an in vivo response). Interestingly, Jacobs et al. (1998) found a decreased responsiveness (increase in intracellular calcium) of human foetal osteoblastic cells to oscillatory and pulsatile fluid flow with increasing frequency, although in a frequency range $(0.5$ to $2 \mathrm{~Hz})$ that is still lower than the $5-10 \mathrm{~Hz}$ "threshold" established by Warden and Turner. More recently, Faure et al. (2008) demonstrated that the expression of vascular endothelial growth factor (VEGF) in osteoblastic cells, which is important for bone angiogenesis, is regulated by stretch frequency. Interestingly, regulation seemed to be different for soluble and matrix-bound isoforms. While expression of soluble VEGF isoforms was found to be upregulated at low $(0.05 \mathrm{~Hz})$ frequencies, the matrix-bound VEGF isoforms was upregulated at high $(5 \mathrm{~Hz})$ frequencies. The implication of these in vitro findings for the in vivo response of bone to loading frequency remains to be investigated.

\section{Vibration studies}

When confronting the results from some of the vibration studies with those of loading studies on individual limbs, it seems hard to explain how such extremely low strains can have an anabolic (and/or antiresorptive) effect. Indeed, many studies on individual limbs have clearly shown that a certain threshold needs to be exceeded to cause a net (positive) change in bone mass. This has led to the idea of error-driven adaptation, meaning that abnormal strains drive bone adaptation, which in its simplest form can be captured by the following equation:

$$
\frac{\mathrm{dm}}{\mathrm{dt}}=\mathrm{C}(\mathbf{r})\left[\phi(\mathbf{r}, \mathrm{t})-\phi_{0}(\mathbf{r})\right]
$$

Where $\mathrm{m}$ is bone mass, $\mathrm{C}$ is a rate constant, $\varphi$ is a strainrelated mechanical stimulus (which is dependent on time and space), $\varphi_{0}$ is a stimulus reference value (threshold), $r$ is a spatial coordinate and $t$ is time. If the stimulus is taken to be strain itself, the threshold is typically of the order of $100-1000 \mu \varepsilon$ in order to induce bone formation. Also, strain (or stimulus) values that cause an effect are typically related to vigorous, abnormal activity, which may only occur a few times per day (Rubin and Lanyon, 1984). Based on the concept that only large, "unusual" strains can have an osteogenic effect, we cannot explain the osteogenic response observed in vibration studies. Alternatively, 
Rubin and co-workers have hypothesised that low magnitude high frequency loading events, which occur multiple times per day throughout the entire skeleton, may be as important for bone architecture as high magnitude, which are related to vigorous activity and which occur only a few times per day (Rubin et al., 2002a). Fritton et al. (2000) quantified the daily strain history for different bones and animals by means of in vivo strain gauge measurements (12-24 hours monitoring) and indeed demonstrated that that large strains (more than $1000 \mu \varepsilon$ ) occur relatively few times a day, while very small strains $(<10 \mu \varepsilon)$ occur thousands of times a day. They could establish a power law relation between frequency and average strain magnitude (during 12 hours) that was similar for the different bones and animals tested:

$$
\varepsilon(\mathrm{f})=\frac{\mathrm{a}}{\mathrm{f}^{\mathrm{b}}}
$$

in which $\mathrm{f}$ is the frequency, $\bar{\varepsilon}(\mathrm{f})$ is the average strain at a certain frequency and $\mathrm{a}$ and $\mathrm{b}$ are constants. Average strain values were quite low, ranging from $1-10 \mu \varepsilon$ at $0.2 \mathrm{~Hz}$ to $0.05-0.5 \mu \varepsilon$ at $10 \mathrm{~Hz}$. By applying vibration for $10-30$ minutes per day at $17-90 \mathrm{~Hz}$ (as in the studies reported here) there will be a "disruption" of the power law relation, shifting it towards (relatively) higher (average) strains for the high frequency loading. Rubin hypothesised that this shift may be responsible for the adaptive (anabolic) response.

A further comparison can be made between the different vibration studies and the studies of Tanaka et al. (2003) and Castillo et al. (2006), who also applied high frequency low magnitude stimulation, although not by means of a vibrating platform, but by means of the (much more controlled) mouse ulna compressive model. Neither of the two studies could find an osteogenic effect of the high frequency signal alone, although strain gauge measurements could clearly demonstrate that high frequency components (up to $50 \mathrm{~Hz}$ ) were transferred to the bone. It is likely that this mechanical stimulation regime disrupted the power law relation of equation (11) and therefore, according to the earlier mentioned hypothesis, this should have led to an osteogenic response. One may speculate why this was not observed and whether it could be related to the shorter daily load application (30 seconds per day versus 10-30 minutes per day for vibration studies), the different wave form (broad frequency noise versus sinusoidal loading for vibration studies) or the different mode of load application (the much more "constrained" axial compression versus "unconstrained" vibration). A final (maybe most important) difference between Tanaka et al. (2003) and Castillo et al. (2006) versus vibration studies was the fact that in the former two studies the low magnitude strains were actually not that low (at least not compared to vibration studies) and reached peak-to-peak values of several hundreds of microstrain.

One may wonder whether bone cells, like osteocytes are able to sense such extremely low strains or the corresponding fluid flows. Again, the theoretical model of You et al. (2001) may suggest some explanation. Interestingly, the strain amplification ratio is predicted to not only increase with increasing load frequency, but also with decreasing load magnitude. Therefore, low-magnitude high frequency bone strains are most efficiently amplified. In the study amplification ratios of 19 and 122 are given for a $1 \mathrm{~Hz}-1000 \mu \varepsilon$ regime and a $20 \mathrm{~Hz}-50 \mu \varepsilon$ regime respectively. The paper does not give an answer to the question whether extrapolation of these data to even higher frequencies and lower bone strains may lead to cellular strains that can be sensed by osteocyte cellular processes. Apart from explanations based on a disruption of the power law relation between frequency and strain magnitude, increased mechanosensitivity or mechanotransduction towards higher frequencies, Rubin and co-workers (Rubin et al., 2002a) and Turner and co-workers (Tanaka et al., 2003; Castillo et al., 2006) have suggested that a phenomenon called stochastic resonance may play a role as well. It refers to the ability of a non-linear system to detect and transmit a weak, otherwise undetectable signal by adding a random signal (such as noise) to it. Castillo et al. (2006) could however not detect such an effect for the investigated combination of sinusoidal carrier signals and added noise signals. At this stage it is unclear whether it could be an explanation for the observed osteogenic effect of vibration (also given the fact that loading regimes applied by vibrating platforms are sinusoidal and therefore not random). At least, based on the sheep studies from Rubin and co-workers (Rubin et al., 2001a, 2001b, 2002a), the effect of vibration does not seem to be systemic, but limited to the limbs that have been mechanically stimulated.

\section{Implant osseointegration studies}

While Rubin and McLeod (1994) found a higher amount of bone ingrowth for a $20 \mathrm{~Hz}$ compared to a $1 \mathrm{~Hz}$ loading regime, De Smet and co-workers found the largest osteogenic effect for the lowest strain rate and frequency (De Smet et al., 2006, 2007). The contradiction may be related to the different modes of load application: while in Rubin and McLeod (1994) the host bone was stimulated, in the studies of De Smet and co-workers the implant was directly loaded. Therefore, it is likely that the strain distribution in the host bone and peri-implant tissues were very different for these loading set-ups. Our group has developed a methodology to create highly accurate, individualised finite element models of bones and implants, based on high-resolution $\mu \mathrm{CT}$ images (Jaecques et al., 2004). This could be a way to evaluate the different local mechanical conditions to which peri-implant tissues are exposed in case of direct versus indirect implant loading. In case load application starts immediately or early after implant installation (as was the case in the studies of De Smet and co-workers), it is important to realise that the mechanical environment will interact with a biological system that can be very different from mature bone tissue. Davies (2003) described peri-implant healing as a process that recapitulates some of the events that also occur during bone fracture healing. It involves blood clot formation and platelet activation, migration, proliferation and differentiation of osteoprogenitor cells, and de novo bone formation. The implant surface characteristics (chemistry, topography) can interact will all of these processes. Given 
these differences compared to mature bone, mechanoregulation of peri-implant healing will rely on mechanisms that are different from adaptation of mature bone. For bone adaptation it was hypothesised that the osteocytes are the mechanosensory cells. In the differentiating tissues around an implant, osteoprogenitor cells surrounded by their provisional matrix may be influenced by mechanical loading. A substantial number of both in vitro and in vivo studies have indeed demonstrated that the differentiation of mesenchymal precursor, progenitor, or stem cells is influenced by mechanical signals (see Estes et al. (2004) for a review). In parallel, computational models have been developed to describe the mechanoregulation of tissue differentiation (Prendergast et al., 1997, Carter et al., 1998, Claes and Heigele, 1999). Our group has validated the model developed by Prendergast and co-workers (Prendergast et al., 1997, Lacroix and Prendergast 2002) for the quantitative description of peri-implant bone formation in an in vivo bone chamber that enables to control the mechanical environment (Geris et al., 2008). An interesting feature of the mechanoregulation model by Prendergast and co-workers is the fact that it treats the differentiating tissues as biphasic (a mixture of solid and fluid constituents). Therefore, when loaded, cells and tissues will be exposed to deformation and fluid flow. In the model, both signals are considered as governing stimuli for tissue differentiation. For a biphasic material deformation and flow will not only depend on the load magnitude, but also the frequency or rate. As such, this model forms an attractive tool to explore how loading rate or frequency may influence tissue differentiation in the gap tissues around an implant.

\section{Concluding remarks}

Mechanical loading is a key factor for bone architecture. Based on the many animal experimental studies that were included in this review, it is clear that loading frequency and rate are important parameters that influence the response to mechanical loading. Much less is however known about the mechanisms that govern the response to loading frequency and rate.

In terms of the effect of loading frequency, results from "high strain" loading studies on individual limbs seem to be in contradiction with results from "low strain" vibration studies. "High strain" individual limb loading studies suggest the existence of an optimal frequency around 5$10 \mathrm{~Hz}$, beyond which loading becomes less osteogenic. "Low strain" vibration studies have reported an osteogenic effect for frequencies within the range of 17-90 Hz. Most of these studies considered only one frequency. The few studies that looked at different frequencies within the same animal model also varied other loading parameters, so that at this moment, it is unclear whether also for vibration studies there may be a frequency "threshold". "High strain" individual limb loading studies that have used broad frequency noise (that contains higher frequency components up to $50 \mathrm{~Hz}$ and higher) could not establish an osteogenic effect. It is unclear whether this apparent discrepancy with respect to high frequency loading is related to the different loading set-ups ("constrained" loading versus "free" vibration) or the fact that mechanotransduction efficiency is different at high strains versus low strains (as suggested by theoretical models that have studied strain amplification from the tissue to the cell level). As strain gauge measurements have demonstrated that high frequency loading components can be transduced to the (cortical) bone for both set-ups, it is unlikely that dampening has been an important confounding factor.

At a more phenomenological level, it has been suggested that the effect of extremely low magnitude high frequency strains may be related to a shift of the power law relation between frequency and strain (equation 11), which may lead to an adaptive response. At a more mechanistic level, theoretical model suggest that high frequency, low amplitude loading may be amplified the most.

Addressing these questions clearly requires further research:

* In order to further explore the difference between "constrained" loading setups (i.e. the loading models that were used in individual limb loading studies) and vibrating platforms, it would be interesting to investigate the effect of both loading modes within the same animal for similar strain magnitudes, loading frequencies and waves. This should be coupled to a thorough mechanical analysis of load transfer in both models, requiring the combination of experimental and computational tools.

* More fundamental research is needed on how load magnitude and frequency influence mechanotransduction. The word mechanotransduction should be interpreted here as the transduction of mechanical signals over multiple length scales (e.g. organ-tissue-cell) as well as the transduction of the mechanical signals into a biochemical response. Again, a combined computational and experimental approach is desirable. Computational models are needed that consider the coupling bone deformation and bone fluid flow (such as poromechanical models, see Cowin, 1999) and/or that are able to bridge the multiple length scales (hierarchical or multiscale models). In vivo and in vitro experiments on the molecular effects of (high frequency, low amplitude) loading should be able to increase our fundamental understanding of the pathways involved.

Extrapolation of bone adaptation studies to mechanoregulation studies of implant osseointegration does not seem to be obvious, given the different biology of peri-implant healing compared to bone adaptation. The number of studies that have looked at the effect of loading frequency and rate on implant osseointegration is still limited, and opposite results have been reported, although for different loading modes (direct versus indirect loading). Again, a number of suggestions for further research can be made:

* The evaluation of direct versus indirect loading within the same animal, in combination with imagebased finite element modelling to quantify the strain distribution, would certainly aid in resolving this apparent contradiction. 
* The effect of loading frequency and rate on tissue differentiation in the peri-implant tissues should be combined with finite element models that consider these tissues as biphasic (or poroelastic). In this way the effect of frequency and rate on fluid flow and possible flow-mediated differentiation can be addressed.

* The effect of vibration (high frequency, extremely low amplitude loading) on osseointegration has not yet been properly addressed. Further in vivo work is needed to fill that gap.

\section{Acknowledgements}

This research was supported by grant OT/04/26 from K.U. Leuven.

\section{References}

Bacabac RG, Smit TH, Mullender MG, Dijcks SJ, Van Loon JJ, Klein-Nulend J (2004) Nitric oxide production by bone cells is fluid shear stress rate dependent. Biochem Biophys Res Commun 315: 823-829.

Burger EH, Klein-Nulend J (1999) Mechanotransduction in bone - role of the lacuno-canalicular network. FASEB J 13: S101-S112.

Burr DB, Martin RB, Schaffler MB, Radin EL (1985) Bone remodeling in response to in vivo fatigue microdamage. J Biomech 18: 189-200.

Carter DR, Beaupre GS, Giori NJ, Helms JA (1998) Stochastic resonance in osteogenic response to mechanical loading. Clin Orthop Relat Res 355: S41-S55.

Castillo AB, Alam I, Tanaka SM, Levenda J, Li J, Warden SJ, Turner CH (2006) Low-amplitude, broadfrequency vibration effects on cortical bone formation in mice. Bone 39: 1087-1096.

Claes LE, Heigele CA (1999) Magnitudes of local stress and strain along bony surfaces predict the course and type of fracture healing. J Biomech 32: 255-266.

Cowin SC (1999) Bone poroelasticity. J Biomech 32: 217-238

Cowin SC (2002) Mechanosensation and fluid transport in living bone. J Musculoskelet Neuronal Interact 2: 256-260.

Davies JE (2003) Understanding peri-implant endosseous healing. J Dent Educ 67: 932-949.

De Smet E, Jaecques SVN, Wevers M, Jansen JA, Jacobs R, Vander Sloten J, Naert IE (2006) Effect of controlled early implant loading on bone healing and bone mass in guinea pigs, as assessed by micro-CT and histology. Eur J Oral Sci 114: 232-242.

De Smet E, Jaecques SV, Jansen JJ, Walboomers F, Vander Sloten J, Naert IE (2007) Effect of constant strain rate, composed of varying amplitude and frequency, of early loading on peri-implant bone (re)modelling. J Clin Periodontol 34: 618-624.

De Souza RL, Matsuura M, Eckstein F, Rawlinson SCF, Lanyon LE, Pitsillides AA (2005) Non-invasive axial loading of mouse tibiae increases cortical bone formation and modifies trabecular organization: A new model to study cortical and cancellous compartments in a single loaded element. Bone 37: 810-818.

Duyck J, Vandamme K, Geris L, Van Oosterwyck H, De Cooman M, Vander Sloten J, Puers R, Naert I (2006) The influence of micro-motion on the tissue differentiation around immediately loaded cylindrical turned titanium implants. Arch Oral Biol 51: 1-9.

Estes BT, Gimble JM, Guilak F (2004) Mechanical signals as regulators of stem cell fate. Curr Top Dev Biol 60: $91-126$.

Faure C, Linossier MT, Malaval L, Lafage-Proust MH, Peyroche S, Vico L, Guignandon A (2008) Mechanical signals modulated vascular endothelial growth factor-A (VEGF-A) alternative splicing in osteoblastic cells through actin polymerization. Bone 42: 1092-1101.

Flieger J, Karachalios T, Khaldi L, Raptou P, and Lyritis G (1998) Mechanical stimulation in the form of vibration prevents postmenopausal bone loss in ovariectomized rats. Calcif Tissue Int 63: 510-514.

Fritton SP, McLeod KJ, Rubin CT (2000) Quantifying the strain history of bone: spatial uniformity and selfsimilarity of low-magnitude strains. J Biomech 33: 317325.

Geris L, Vandamme K, Naert I, Vander Sloten J, Duyck J, Van Oosterwyck H (2008) Application of mechanoregulatory models to simulate peri-implant tissue formation in an in vivo bone chamber. J Biomech 41: 145154.

Gross TS, Srinivasan S, Liu CC, Clemens TL, Bain SD (2002) Noninvasive loading of the murine tibia: an in vivo model for the study of mechanotransduction. J Bone Miner Res 17: 493-501.

Hsieh YF, Wang T, Turner CH (1999) Viscoelastic response of the rat loading model: implications for studies of strain-adaptive bone formation. Bone 25: 379-382.

Hsieh YF, Turner CH (2001) Effects of loading frequency on mechanically induced bone formation. J Bone Miner Res 16: 918-924

Huiskes R, Weinans H, van Rietbergen B (1992) The relationship between stress shielding and bone resorption around total hip stems and the effects of flexible materials. Clin Orthop Relat Res 274: 124-134.

Huiskes R, Van Driel WD, Prendergast PJ, Søballe K (1997) A biomechanical regulatory model for periprosthetic fibrous-tissue differentiation. J Mater Sci Mater Med 8: 785-788.

Jacobs CR, Yellowley CE, Davis BR, Zhou Z, Cimbala JM, Donahue HJ (1998) Differential effect of steady versus oscillating flow on bone cells. J Biomech 31: 969-976.

Jaecques SV, Van Oosterwyck H, Muraru L, Van Cleynenbreugel T, De Smet E, Wevers M, Naert I, Vander Sloten J (2004) Individualised, micro CT-based finite element modelling as a tool for biomechanical analysis related to tissue engineering of bone. Biomaterials 25: 1683-1696.

Judex S, Gross TS, Zernicke RF (1997) Strain gradients correlate with sites of exercise-induced bone-forming surfaces in the adult skeleton. J Bone Miner Res 12: 17371745. 
Kaspar D, Seidl W, Neidlinger-Wilke C, Beck A, Claes L, Ignatius A (2002) Proliferation of human-derived osteoblast-like cells depends on the cycle number and frequency of uniaxial strain. J Biomech 35: 873-880.

Kotha SP, Hsieh YF, Strigel RM, Muller R, Silva MJ (2004) Experimental and finite element analysis of the rat ulnar loading model-correlations between strain and bone formation following fatigue loading. J Biomech 37: 541548 .

Lacroix D, Prendergast PJ (2002) A mechanoregulation model for tissue differentiation during fracture healing: analysis of gap size and loading. J Biomech 35: 1163-1171.

LaMothe JM, Zernicke RF (2004) Rest insertion combined with high-frequency loading enhances osteogenesis. J Appl Physiol 96: 1788-1793.

LaMothe JM, Hamilton NH, Zernicke RF (2005) Strain rate influences periosteal adaptation in mature bone. Med Eng Phys 27: 277-284.

Lanyon LE, Rubin CT (1984) Static vs dynamic loads as an influence on bone remodelling. J Biomech 17: 897905.

Lee KC, Maxwell A, Lanyon LE (2002) Validation of a technique for studying functional adaptation of the mouse ulna in response to mechanical loading. Bone 31: 407412.

Liskova M, Hert J (1971) Reaction of bone to mechanical stimuli. 2. Periosteal and endosteal reaction of tibial diaphysis in rabbit to intermittent loading. Folia Morphol (Praha) 19: 301-317.

Mosley JR, March BM, Lynch J, Lanyon LE (1997) Strain magnitude related changes in whole bone architecture in growing rats. Bone 20: 191-198.

Mosley JR, Lanyon LE (1998) Strain rate as a controlling influence on adaptive modeling in response to dynamic loading of the ulna in growing male rats. Bone 23: 313-318.

Oxlund BS, Ortoft G, Andreassen TT, Oxlund H (2003) Low-intensity, high-frequency vibration appears to prevent the decrease in strength of the femur and tibia associated with ovariectomy of adult rats. Bone 32: 69-77.

Parfitt AM, Drezner MK, Glorieux FH, Kanis JA, Malluche H, Meunier PJ, Ott SM, Recker RR (1987) Bone histomorphometry: standardization of nomenclature, symbols, and units. Report of the ASBMR Histomorphometry Nomenclature Committee. J Bone Miner Res 2: 595-610.

Pilliar RM, Cameron HU, Binnington AG, Szivek J, Macnab I (1979) Bone ingrowth and stress shielding with a porous surface coated fracture fixation plate. J Biomed Mater Res 13: 799-810.

Prendergast PJ, Huiskes R, Søballe K (1997) ESB Research Award 1996. Biophysical stimuli on cells during tissue differentiation at implant interfaces. J Biomech 30: 539-548.

Robling AG, Burr DB, Turner CH (2001) Recovery periods restore mechanosensitivity to dynamically loaded bone. J Exp Biol 204: 3389-3399.

Rubin CT and Lanyon LE (1985) Regulation of bone mass by mechanical strain magnitude. Calcif Tissue Int 37: 411-417.
Rubin CT, McLeod KJ (1994) Promotion of bony ingrowth by frequency-specific, low-amplitude mechanical strain. Clin Orthop Relat Res 298: 165-174.

Rubin C, Turner AS, Bain S, Mallinckrodt C, McLeod K (2001a) Anabolism. Low mechanical signals strengthen long bones. Nature 412: 603-604.

Rubin C, Xu G, Judex S (2001b) The anabolic activity of bone tissue, suppressed by disuse, is normalized by brief exposure to extremely low-magnitude mechanical stimuli. FASEB J 15: 2225-2229.

Rubin C, Turner AS, Mallinckrodt C, Jerome C, McLeod K, Bain S (2002a) Mechanical strain, induced noninvasively in the high-frequency domain, is anabolic to cancellous bone, but not cortical bone. Bone 30: 445452.

Rubin C, Turner AS, Muller R, Mittra E, McLeod K, Lin W, Qin YX (2002b) Quantity and quality of trabecular bone in the femur are enhanced by a strongly anabolic, noninvasive mechanical intervention. J Bone Miner Res 17: 349-357.

Søballe K, Hansen ES, Rasmussen H, Jorgensen PH, Bunger C (1992) Tissue ingrowth into titanium and hydroxyapatite-coated implants during stable and unstable mechanical conditions. J Orthop Res 10: 285-299.

Srinivasan S, Weimer DA, Agans SC, Bain SD, Gross TS (2002) Low-magnitude mechanical loading becomes osteogenic when rest is inserted between each load cycle. J Bone Miner Res 17: 1613-1620.

Tanaka SM, Alam IM, Turner CH (2003) Stochastic resonance in osteogenic response to mechanical loading. FASEB J 17: 313-314.

Torrance AG, Mosley JR, Suswillo RF, Lanyon LE (1994) Noninvasive loading of the rat ulna in vivo induces a strain-related modeling response uncomplicated by trauma or periostal pressure. Calcif Tissue Int 54: 241247.

Turner CH, Forwood MR, Otter MW (1994) Mechanotransduction in bone: do bone cells act as sensors of fluid flow? FASEB J 8: 875-878.

Turner SH, Owan I, Takano Y (1995) Mechanotransduction in bone: role of strain rate. Am J Physiol 269: 438-442.

Turner CH (1998) Three rules for bone adaptation to mechanical stimuli. Bone 23: 399-407.

Vandamme K, Naert I, Geris L, Vander Sloten J, Puers R, Duyck J (2007) Influence of controlled immediate loading and implant design on peri-implant bone formation. J Clin Periodontol 34: 172-181.

Warden SJ, Turner CH (2004) Mechanotransduction in the cortical bone is most efficient at loading frequencies of 5-10 Hz. Bone 34: 261-270.

Xie L, Jacobson JM, Choi ES, Busa B, Donahue LR, Miller LM, Rubin CT, Judex S (2006) Low-level mechanical vibrations can influence bone resorption and bone formation in the growing skeleton. Bone 39: 10591066.

You L, Cowin SC, Schaffler MB, Weinbaum S (2001) A model for strain amplification in the actin cytoskeleton of osteocytes due to fluid drag on pericellular matrix. J Biomech 34: 1375-1386. 


\section{Discussion with Reviewers}

L. Vico: As for whole body vibration, the "Rubin's school" claimed for signals below $1 \mathrm{~g}$ which is not the case for other studies of other teams who have tested accelerations greater than $1 \mathrm{~g}$. What do the authors think about the acceleration level?

Authors: The specific parameter settings in Whole Body Vibration studies that result in the strongest anabolic bone response are still a matter of debate. The identification of an acceleration level leading to an "optimal" strain environment able to induce bone cell response is a complex issue. As discussed in this paper, Whole Body Vibration studies often report on the acceleration level and frequency of the vibration loading; these are not independent parameters, but linked through the displacement amplitude of the platform (equation 9). It also has to be realized that the relationships between local tissue strains and global loading are only poorly understood, since force transfer through the limbs may involve soft tissue dampening and muscle activation (see introductory paragraph of the "Bone adaptation studies" section). Hence, more fundamental research is required for analyzing first, the transmission of this acceleration level from the body level through the organ level and tissue level down to the cell level, and second, to further enhance our knowledge on how the local mechanical environment around the cell affects cell response.

Extremely low-level $(<1 \mathrm{~g})$ high-frequency vibrations were found by the Rubin's school as the "optimal" combination for promoting bone response, whereas other studies (Flieger et al., 1998; Oxlund et al., 2003) reported (relatively) higher magnitude (2-3 g) high-frequency vibrations to be osteogenic. One may speculate that these different combinations of parameters would induce similar strain environments at the cell level. Indeed, You et al. (2001) in their mathematical model affirmed that higher "amplification ratios" (i.e., ratio of cellular strain versus tissue strain, see also second subparagraph in the "Discussion" section) were obtained for lower tissue strains (evaluated by You et al. down to $50 \mu \varepsilon$ ) and higher frequencies (evaluated up to $20 \mathrm{~Hz}$ ). Hence, it may be that acceleration levels, either smaller than $1 \mathrm{~g}$ (causing tissue strains of the order of 1-10 $\mu \varepsilon$., as quantified by Rubin and co-workers) or larger than $1 \mathrm{~g}$ (2-3 g, and therefore expected to causing larger tissue strains) would generate similar cellular strain levels and therefore similar osteogenic responses.

L. Vico: One important difference versus free vibrations might be the fact that controlled loading studies in turkey, rat, or mouse limb bones mainly showed a cortical modelling response while whole body vibrations seems to alter primarily the trabecular compartment. Do the authors have an explanation or a hypothesis?

Authors: Loading studies on individual limbs have shown that relatively high magnitude (order of $1000 \mu \varepsilon$ ) loading is needed for a net positive change in cortical bone mass. The studies reviewed in our paper simply did not investigate the trabecular bone response (among others because the applied animal loading models were not suitable for this) and therefore they do neither confirm nor reject the hypothesis that also trabecular bone responds to such stimuli. Indeed, De Souza et al. (2005) developed a non-invasive axial loading model in the mouse tibia, which enabled them to study both cortical and trabecular bone response in the same limb. They demonstrated that mechanical loading induced magnitude-related increase in bone cortical formation (for cortical strains between 1150 and $2000 \mu \varepsilon$ ) and changes in trabecular bone architecture in mice.

For their sheep experiments, Rubin and co-workers only found a response to whole body vibration in trabecular bone, and not in cortical bone (Rubin et al., 2001a, 2002a, 2002b). However, other vibration studies did report a cortical bone response as well. Xie et al. (2006) demonstrated that $45 \mathrm{~Hz}-0.3 \mathrm{~g}$ vibration affected bone formation rates $(\mathrm{BFR} / \mathrm{BS})$ on the endocortical surface of the metaphysis in the proximal tibia of 8-week-old BALB/ cByJ mice (in addition to trabecular bone effects). Judex et al. (2007) showed that $90 \mathrm{~Hz}-0.15 \mathrm{~g}$ vibration enhanced bone formation rates in the proximal tibia of OVX rats for both cortical and trabecular bone. These findings do not support the hypothesis that whole body vibration only affects trabecular bone mass.

Cortical and trabecular bone present very distinct architectures, and therefore it is likely that the same externally applied loading regime will result in different mechanical stimuli at tissue and cell level. In addition, trabecular bone may be metabolically more active by virtue of a larger specific surface area that is available for modeling and remodeling. Architectural, mechanical and biological differences between cortical and trabecular bone may be related to a different response to the same mechanical loading regime.

M. Bohner: A question related to the non-linear effect of loading frequency: since load signaling to bone cells depends on the fluid flow in canaliculi, and as the size of these canaliculi is likely to be known, is there no article attempting to calculate the fluid flow as a function of loading frequency and amplitude? (is that what the authors suggest in the second last sub-paragraph?)

Authors: A non-linear effect of loading frequency on bone response was reported in the controlled loading study of Warden and Turner (2004). They found bone adaptation to increase with increasing loading frequency up to 5-10 $\mathrm{Hz}$, while a decrease was found for higher frequencies. They hypothesized that with frequencies higher than 10 $\mathrm{Hz}$, the mechanotransduction process becomes less efficient, leading to reduced cellular stimulation and a resultant reduction in bone formation.

To our knowledge, no experimental study has been performed that directly measured the load-induced fluid flow in the canaliculi. Alternatively, mathematical models have been developed with the aim to understand the mechanical stimuli experienced by bone cells. In these models the fluid flow in the canaliculi is assumed to follow Darcy's law, meaning that fluid velocity is related to the pressure gradient along the canaliculi (Weinbaum et al., 1994; Zeng et al., 1994). In one study, Zeng and coworkers (1994) attempted to couple the small-scale fluid mechanics 
with the larger scale deformation of the entire osteon, when assuming bone as a poroelastic material. The canalicular geometry was idealized to a tube containing a centrally positioned osteocyte process. The velocity profile in the fluid annulus and the shear stresses acting at the exterior surface of the osteocytic process were derived from the pressure gradients generated by a sinusoidal loading applied to the osteon. You et al. (2001) improved this model by including pericellular matrix fibers anchoring and centering the cell process in its canaliculus and in turn coupling them to the intracellular actin cytoskeleton. The mechanical signal provided by the drag forces exerted on these fibers and transmitted to the cell process, appeared to be more important than shear forces on the cell membrane in stimulating and activating the cellular response. As discussed in our paper You et al. investigated the effect of loading frequency, but did not find a theoretical evidence for the hypothesis that the mechanotransduction process becomes less efficient for frequencies higher than $10 \mathrm{~Hz}$.

The limitations of this mathematical model need to be mentioned. The geometry of the lacuno-canalicular network was idealized to a single canaliculus that drains towards the Haversian canal and, in addition, the annular region between the cell process and the canalicular wall was modeled as a cylindrical canal. The knowledge on the actual geometry with all its "irregularities" is indeed critical for estimating the flow in the canaliculi, as demonstrated by Anderson and Knothe Tate (2008). In their computational model the actual geometry of the pericellular space was modeled, based on transmission electron micrographs (TEM), and magnitude and spatial variation of fluid velocity, pressure as well as shear stresses on the cell surface were predicted. Although the dependency of the fluid profile in canaliculi on loading frequency was not investigated, this study has the merit to evaluate the effect of the idealization of the pericellular fluid space geometry and dimensions. Indeed, the study demonstrated that this idealization underestimated fluid pressures, flow velocities as well as shear stresses. However, although this study took into account more complex situations from a geometrical point of view, it neglected all the compliant cell structures (cytoskeleton, cell membrane, transmembrane proteins and cytosol), which may be very important for mechanotransduction.

\section{Additional References}

Anderson EJ, Knothe Tate ML (2008). Idealization of pericellular fluid space geometry and dimension results in a profound underprediction of nano-microscale imparted by fluid drag on osteocytes. J Biomech 41: 1736-1746.

Weinbaum S, Cowin SC, Zeng Y (1994). A model for the excitation of osteocytes by mechanical loading induced bone fluid shear stresses. J Biomech 273: 339-360.

Zeng Y, Cowin SC, Weinbaum S (1994). A fiber matrix model for fluid flow and streaming potentials in the canaliculi of an osteon. Ann Biomed Eng 22: 280-292. 